\title{
NOVEL LOW TEMPERATURE SUPERCONDUCTING BOLOMETERS
}

\author{
M. Nahum and P. L. Richards \\ Departments of Physics, University of California at Berkeley, \\ and Materials Sciences Division, Lawrence Berkeley Laboratory, \\ Berkeley, CA 94720
}

M. Gaidis and D. E. Prober

Department of Applied Physics, Yale University,

New Haven, CT 06520-2157

LBL- -33349

DE93 010433

* This work was supported in part by the Director, Office of Energy

Research, Office of Basic Energy Sciences, Materials Sciences Division of the

U. S. Department of Energy under Contract No. DE-AC03-76-SF00098. 


\title{
NOVEL LOW TEMPERATURE SUPERCONDUCTING BOLOMETERS
}

\author{
M. Nahum and P. L. Richards \\ Departments of Physics, University of California at Berkeley, \\ and Materials Sciences Division, Lawrence Berkeley Laboratory, \\ Berkeley, CA 94720 \\ M. Gaidis and D. E. Prober \\ Department of Applied Physics, Yale University, \\ New Haven, CT 06520-2157
}

\begin{abstract}
We have designed and fabricated novel antenna-coupled superconducting bolometers for submillimeter and millimeter waves, which make use of the thermal boundary resistance between metals and insulators and the trapping of quasiparticles at metal-superconductor interfaces. We have used these devices to make measurements of the frequency response and optical efficiency of the log-periodic antennas between $90 \mathrm{GHz}$ to $1.8 \mathrm{THz}$.

\section{OPERATING PRINCIPLES}

The low- $T_{\mathfrak{c}}$ microbolometer consists of a thin strip of superconductor whose temperature is regulated at the midpoint of its resistive transition. The strip serves both as the resistive load to thermalize the infrared current from a planar lithographed antenna, and as a sensitive thermometer to measure the resulting temperature rise. Thermal isolation is provided by the thermal boundary resistance between the strip and the dielectric substrate, and by the reflection of quasiparticles at the interface between the transition edge thermometer and the superconducting antenna. All of the components are deposited directly on the substrate and can be produced in arrays using standard photolithographic techniques. The thermally active volume of this bolometer is $\approx 1 \mu \mathrm{m}^{3}$ compared with $\approx 10^{8} \mu \mathrm{m}^{3}$ for more conventional millimeter wave bolometers. A detailed review of the low- $T_{c}$ microbolometer is given in Ref. 1 .
\end{abstract}

\section{FABRICATION AND ELECTRICAL CHARACTERIZATION}

The prototype devices were fabricated on fused quartz substrates using conventional photolithographic techniques. Tantalum films $(\approx 500 \AA$ thick) were sputtered on $\approx 50 \AA$ niobium buffer layers to nucleate the growth of the bcc-phase of tantalum $\left(T_{c} \approx 4.0 \mathrm{~K}\right)$, and subsequently patterned by liftoff to define a strip with length $l=6 \mu \mathrm{m}$ and width $\mathrm{w}=2 \mu \mathrm{m}$. The surface of the strip was ion milled after which $2500 \AA$ of niobium $\left(T_{c}=9.2 \mathrm{~K}\right.$ ) was sputtered and patterned by liftoff to define a log-periodic antenna. The substrate was then mounted onto the back side of a hemispherical crystal quartz lens with a thin layer of grease, and electrical contacts were made at the antenna terminals with silver paint. The substrate-lens combination was temperature regulated at the center of the resistive transition of the tantalum strip using a commercial germanium resistance thermometer and controller.

The dc resistance of the tantalum strip was $4 \Omega$ at the center of the resistive transition and the temperature coefficient of resistance was $\mathrm{dR} / \mathrm{dT}=50 \Omega / \mathrm{K}$. The electrical responsivity of the bolometer, determined from the dc current-voltage characteristics when temperature regulated at the center of the resistive transition, was $10^{4} \mathrm{~V} / \mathrm{W}$ at a bias current of $10 \mu \mathrm{A}$. The time constant of the device, nteasured with a $90 \mathrm{GHz}$ Gunn oscillator whose bias was electrically modulated, was less than $1 \mu \mathrm{s}$. The total noise of the biased bolometer was $0.5 \mathrm{nVHz}^{-1 / 2}$ which yields an electrical noise equivalent power (NEP) of $5 \times 10^{-14} \mathrm{WHz}^{-1 / 2}$ at $100 \mathrm{~Hz}$ modulation frequency, indicating that the sensitivity was limited by the intrinsic energy fluctuations in the active region. The electrical and thermal properties of this detector are in excellent agreement with the predicted performance [1]. We are currently fabricating microbolometers that use the $\mathrm{Ti}\left(\mathrm{T}_{\mathrm{c}}=0.35 \mathrm{~K}\right)$ as the transition edge thermometer/absorber. The NEP of the titanium microbolometer is expected to be $\approx 3 \times 10^{-17} \mathrm{WHz}^{-1 / 2}$, this is almost a factor of 10 better than the best available direct detectors of submilimeter and millimctcr waves, when operated at ${ }^{3} \mathrm{He}$ same temperatures. 
FREQUENCY DEPENDENT OPTICAL EFFICIENCY OF LOG-PERIODIC ANTENNAS

In order to measure the intrinsic frequency response of the antenna, it is necessary that the mpedance of the sensor which terminates the antenna not vary significantly within the frequency range of interest, and in addition, the sensitivity of the device should be compatible with the measurement technique. The low- $\mathrm{T}_{\mathrm{c}}$ microbolometer satisfies both of these requirements [1].

The frequency response of the antenna was measured with a Fourier transform infrared spectrometer (FTIR). The optical system consisted of an off-axis ellipsoidal mirror that was used to collimate the beam onto the quartz lens (diameter $=1.3 \mathrm{~cm}$ ), whose center coincides with one of the focal points. A light pipe was used to feed the radiation from the FTIR to the detector, and was terminated at the second focal point of the mirror. The power spectrum of the incident radiation was determined by replacing the microbolometer with a conventional composite bolometer (operated at $\mathrm{T}=2.0 \mathrm{~K}$ ) whose absorbing element was a bismuth film. The normalized antenna response is shown in Fig. 1. The frequencies of the vertical arrows are spaced by factors of $\tau^{-1 / 2}=1.41$, where $\tau=0.5$ is the periodicity factor which is appropriate for this particular antenna structure [2j. The agreement between the predicted and observed periodicity is almost perfect up to the 6th peak at $590 \mathrm{GHz}$, indicating that the antenna pattern is being modulated with the logarithmic periodicity of the antenna structure. The fit is less

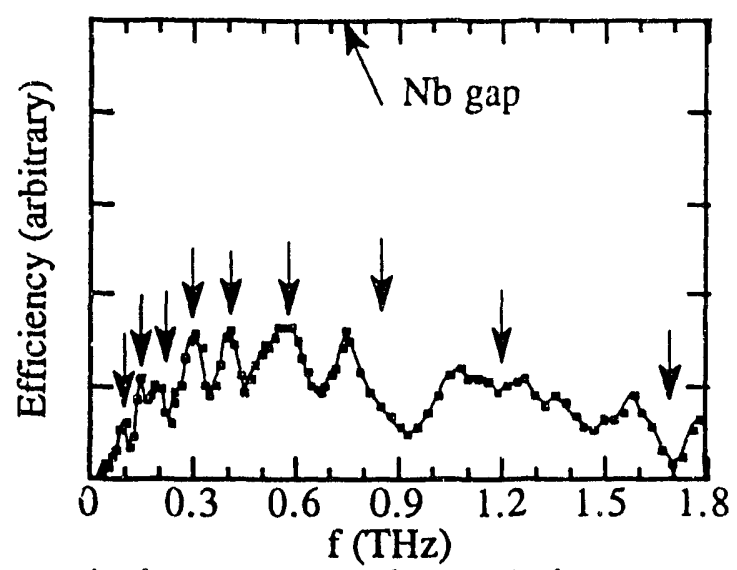

Fig. 1: The frequency dependent optical response of a log-periodic antenna. The logarithmic periodicity resulting from the antenna structure is indicated by the set of vertical arrows.

good for predicted peaks above the energy gap of $\mathrm{Nb}$ at $720 \mathrm{GHz}$, but significant response extends up to $2 \mathrm{THz}$. We believe that the high frequency limit is not limited by the intrinsic properties of the antenna, but rather due to absorption losses in the dielectric lens and substrate.

In order to accurately measure the coupling efficiency, it is necessary to have a well calibrated source. In addition, the beam patterns of both the source and the detector must be matched to avoid coupling losses. The optical configuration discussed here, is ideally suited for this measurement, when a blackbody source is placed at the second focus of the mirror. Our measurements indicate an integrated antenna efficiency of $\approx 20 \%$ between $90-720 \mathrm{GHz}$, when corrected for the impedance mismatch between the absorber $(\approx 4 \Omega)$ and antenna $(120 \Omega)$ impedances.

\section{CONCLUSIONS}

In conclusion, our measurements demonstrate an optical efficiency of $\approx 20 \%$ for the a planar lithographed antenna, with a response which is relatively independent of frequency up to $2 \mathrm{THz}$. We have also demonstrated that the superconducting $\mathrm{Nb}$ antenna can be operated above its energy gap withuut significant additional losses.

This work was supported by Director, Office of Energy Research, Office of Basic Energy Sciences, Materials Sciences Division of the U. S. Department of Energy under Contract No. DE-ACO3$76 S F 00098$.

[1] J. Mees, M. Nahum, and P. L. Richards, Appl. Phys. Lett. 59, 2329 (1991).

[2] R. H DuHamel and D. E. Isbell, IRE National Convention Record, Part I, pp. 119-128, 1957. 

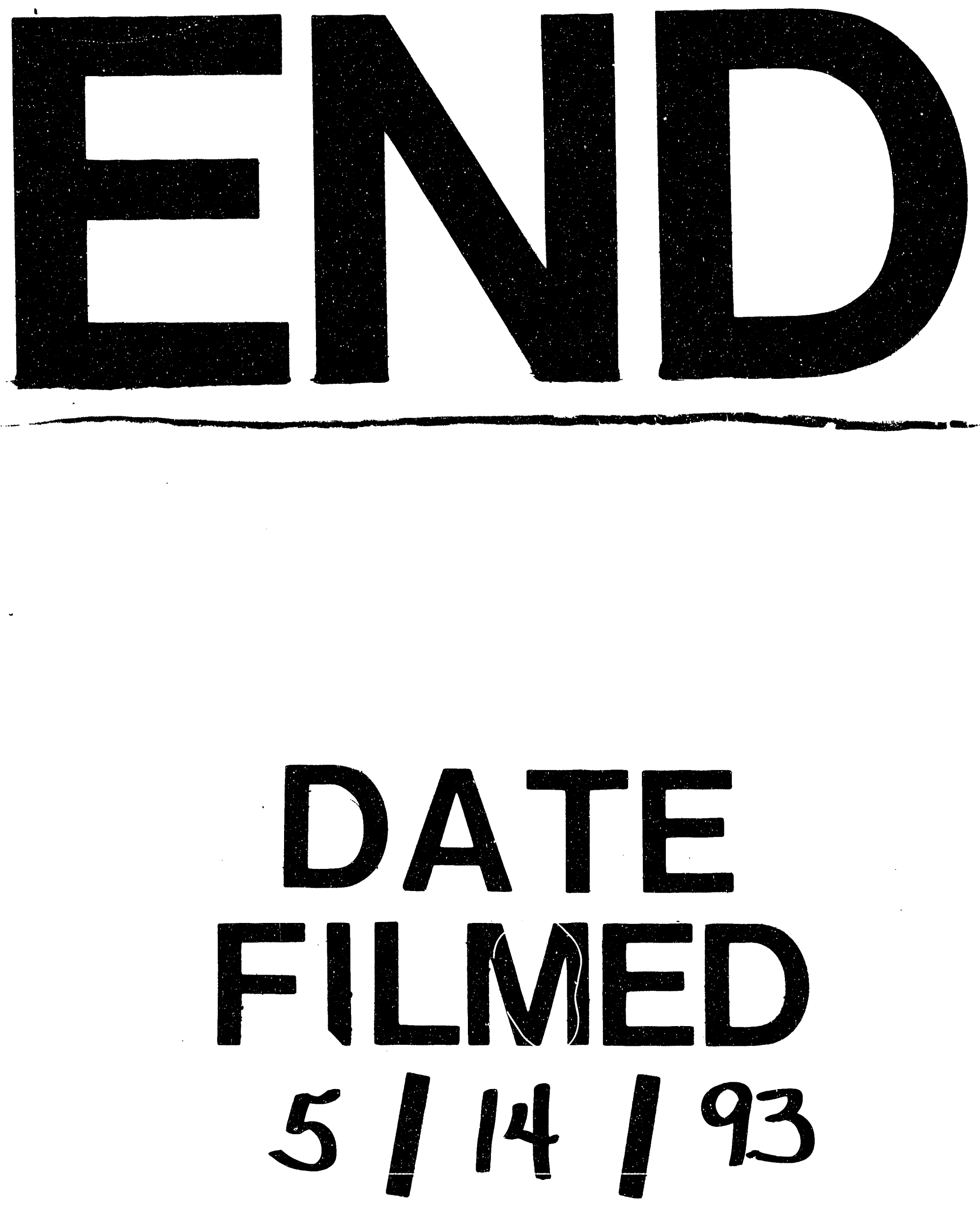
